\title{
Optical Bell-state analysis in the coincidence basis
}

\author{
S. P. Walborn. . W. A. T. Nogueira, S. Pádua, and C. H. Monken \\ Universidade Federal de Minas Gerais, Caixa Postal 702, Belo Horizonte, MG 30123-970, Brazil
}

(Dated: October 29, 2018)

\begin{abstract}
Many quantum information protocols require a Bell-state measurement of entangled systems. Most optical Bell-state measurements utilize two-photon interference at a beam splitter. By creating polarization-entangled photons with spontaneous parametric down-conversion using a first-order Hermite-Gaussian pump beam, we invert the usual interference behavior and perform an incomplete Bell-state measurement in the coincidence basis. We discuss the possibility of a complete Bell-state measurement in the coincidence basis using hyperentangled states [Phys. Rev. A, 58, R2623 (1998)].
\end{abstract}

PACS numbers: 03.67.-a, 03.67.Hk, 42.50.-p

Many quantum information schemes require entangled Bell-states as a resource. Furthermore, protocols such as dense coding [1], 2], quantum teleportation [3, 4, 5] and entanglement swapping [3, 6, 7] require a Bell-state measurement (BSM), that is, distinguishing between the four Bell-states:

$$
\begin{aligned}
& \left|\psi^{ \pm}\right\rangle=\frac{1}{\sqrt{2}}\left(|h\rangle_{1}|v\rangle_{2} \pm|v\rangle_{1}|h\rangle_{2}\right), \\
& \left|\phi^{ \pm}\right\rangle=\frac{1}{\sqrt{2}}\left(|h\rangle_{1}|h\rangle_{2} \pm|v\rangle_{1}|v\rangle_{2}\right),
\end{aligned}
$$

where $\left|\psi^{-}\right\rangle$is the antisymmetric singlet state and $\left|\psi^{+}\right\rangle,\left|\phi^{ \pm}\right\rangle$are the symmetric triplet states. In this paper we use polarization Bell-states, so $h$ and $v$ stand for horizontal and vertical polarization and 1 and 2 represent different spatial modes. These states are easily generated using spontaneous parametric down-conversion [8, 9]. However, difficulty arises when one wants to distinguish between the four Bell-states. In fact, it has been proven that a complete Bell-state measurement (discriminating between all four states with $100 \%$ efficiency) using only linear optics is impossible 10, 11, 12, 13. A complete BSM was realized in ref. [5], but the entangled systems were two degrees of freedom of the same photon. Using nonlinear optical processes, one can discriminate among the four states with low efficiency 14. It is possible to perform an incomplete BSM of polarizationentangled photons using two-photon interference and polarizing beam splitters [2, 15]. Another possible scheme is using photon absorption in properly prepared atoms [16. Using hyperentangled states, a complete Bell-state measurement is possible [17], though this scheme, along with those used in [2, 18 requires detectors sensitive to photon number. For momentum entanglement, there are several methods that allow one to distinguish two [18, 19] of the four states and yet another that discriminates between all four with $25 \%$ efficiency [19].

Expanding on previous results [2 17, we wish to report on methods for optical Bell-state measurement in

* swalborn@fisica.ufmg.br
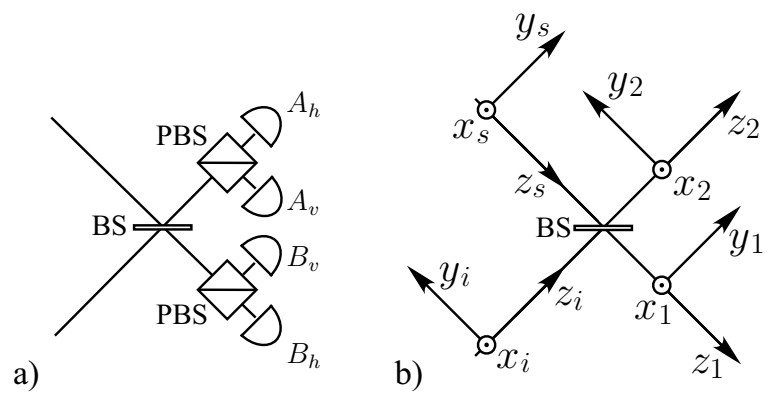

FIG. 1: a) Incomplete Bell-state analyzer. PBS are polarizing beam splitters at $0^{\circ}$. b) Two-photon interference at a beam splitter (BS). Photons $s$ and $i$ pass through the BS into modes 1 and 2 .

the coincidence basis, that is, each photon triggering a different detector. We use polarization-entanglement due to the ease with which one can generate and manipulate polarization-entangled photon pairs.

\section{INCOMPLETE BELL-STATE ANALYSIS IN THE COINCIDENCE BASIS}

Most optical BSM's [2, 15 of polarization-entangled photons rely on Hong-Ou-Mandel type two-photon interference at a 50-50 beam splitter [20]. Consider the Bell-state analyzer (BSA) shown in fig. 1 la, such as the one used in the experimental demonstration of dense coding [2]. This BSA is capable of separating the four Bell states into 3 classes, resulting in a "trit" ( $\approx 1.58$ bits) of transmitted information. A 50-50 beam splitter is used to separate $\left|\psi^{-}\right\rangle$from $\left|\psi^{+}\right\rangle,\left|\phi^{ \pm}\right\rangle$: bosonic symmetry requires that photons in $\left|\psi^{-}\right\rangle$end up in different outputs while photons in $\left|\psi^{+}\right\rangle,\left|\phi^{ \pm}\right\rangle$end up in the same output 21. With the polarizing beam splitters (PBS) separating $h$ and $v$ polarizations, coincidences at $A_{h} B_{v}$ or $A_{v} B_{h}$ are the signature of $\left|\psi^{-}\right\rangle$. The PBS separate $\left|\psi^{+}\right\rangle$from $\left|\phi^{ \pm}\right\rangle$: coincidences at $A_{h} A_{v}$ or $B_{h} B_{v}$ are characteristic of $\left|\psi^{+}\right\rangle$. For $\left|\phi^{ \pm}\right\rangle$, we have two photons at $A_{h} A_{h}, A_{v} A_{v}$, $B_{h} B_{h}$ or $B_{v} B_{v}$. Thus, detection of $\left|\psi^{-}\right\rangle,\left|\psi^{+}\right\rangle$and $\left|\phi^{ \pm}\right\rangle$ requires detectors capable of distinguishing between one 
and two photons. Such detectors are presently available, however they suffer from low efficiencies and/or high dark counts [22, 23, 24]. As mentioned in refs. [2, 18], this problem can be partially solved by replacing each detector with two detectors and an additional 50-50 beam splitter. This enables one to detect only half of the twophoton occurrences and increases the complexity of the detection system, since an eight detector system is necessary.

We could avoid this requirement on the detectors if we could invert the interference behavior: photons in the triplet (singlet) states go to different (the same) detectors and can then be further discriminated by the PBS. This can be achieved by generating polarizationentangled photons using an antisymmetric pump beam, such as the first-order Hermite-Gaussian beam $\mathrm{HG}_{01}$, as we will show below. Then, pumping with $\mathrm{HG}_{01},\left|\psi^{-}\right\rangle$ results in two photons in either output port. Since the two photons are orthogonally polarized, coincidences at detectors $A_{h} A_{v}$ or $B_{h} B_{v}$ are the signature of $\left|\psi^{-}\right\rangle$. The states $\left|\psi^{+}\right\rangle$and $\left|\phi^{ \pm}\right\rangle$give one photon in each output port. Since the photon pairs of $\left|\psi^{+}\right\rangle$are orthogonally polarized, $\left|\psi^{+}\right\rangle$gives coincidence counts at detectors $A_{h} B_{v}$ or $B_{h} A_{v} .\left|\phi^{ \pm}\right\rangle$results in coincidence counts at $A_{h} B_{h}$ or $A_{v} B_{v}$. All detector combinations identifying the three cases $\left|\psi^{+}\right\rangle,\left|\psi^{-}\right\rangle$and $\left|\phi^{ \pm}\right\rangle$correspond to coincidences at different detectors. Let us now discuss multimode interference and show how an antisymmetric pump beam inverts the interference behavior. A two-mode Bell-state, as given by Eq. (2), incident on a beam splitter as shown in fig. $1 \mathrm{~b} \mathrm{~b}$, must preserve its overall bosonic character. Thus if the polarization component of the state is symmetric (antisymmetric), then the spatial component must also be symmetric (antisymmetric) [21]. However, if we consider multimode fields, we must also take into account the transverse spatial properties of the two-photon state as additional degrees of freedom. The combined transverse-spatial and polarization symmetry of the twophoton wave packet determines whether the fields will interfere constructively or destructively. Here we consider photon pairs created by spontaneous parametric downconversion (SPDC) incident on opposite inputs of a beam splitter 20, 25]. A field reflected by the beam splitter undergoes a reflection in the horizontal $(y)$ direction, while a transmitted field does not suffer any reflection, as illustrated in fig. 1]b.

Using the standard theory of SPDC[26, 27], it can be shown that the multimode coincidence-detection amplitudes of the polarization Bell-states in the two outputs of a balanced HOM interferometer are given by [25]

$$
\begin{aligned}
\mathbf{\Psi}^{ \pm}\left(\mathbf{r}_{1}, \mathbf{r}_{2}\right)= & \exp \left\{\frac{i K}{2 Z}\left[\left(x_{1}-x_{2}\right)^{2}+\left(y_{1}-y_{2}\right)^{2}\right]\right\} \times \\
& {\left[\mathcal{W}\left(\frac{x_{1}+x_{2}}{2}, \frac{y_{1}+y_{2}}{2}, Z\right) \mp \mathcal{W}\left(\frac{x_{1}+x_{2}}{2}, \frac{-y_{1}-y_{2}}{2}, Z\right)\right](\mathbf{h v} \pm \mathbf{v h}), } \\
\boldsymbol{\Phi}^{ \pm}\left(\mathbf{r}_{1}, \mathbf{r}_{2}\right)= & \exp \left\{\frac{i K}{2 Z}\left[\left(x_{1}-x_{2}\right)^{2}+\left(y_{1}-y_{2}\right)^{2}\right]\right\} \times \\
& {\left[\mathcal{W}\left(\frac{x_{1}+x_{2}}{2}, \frac{y_{1}+y_{2}}{2}, Z\right)-\mathcal{W}\left(\frac{x_{1}+x_{2}}{2}, \frac{-y_{1}-y_{2}}{2}, Z\right)\right](\mathbf{h h} \pm \mathbf{v v}), }
\end{aligned}
$$

where $\Psi^{ \pm}\left(\boldsymbol{\Phi}^{ \pm}\right)$is the coincidence-detection amplitude for $\left|\psi^{ \pm}\right\rangle\left(\left|\phi^{ \pm}\right\rangle\right)$. $\mathcal{W}(x, y, Z)$ is the transverse amplitude profile of the pump beam (propagated from $z=0$ to $z=Z$ ) transferred to the coincidence-detection amplitude [26], $K$ is the magnitude of the pump beam wave vector, and $\mathbf{h}$ and $\mathbf{v}$ are unit polarization vectors in the $h$ - and $v$-directions, respectively. The vectors $\mathbf{r}_{1}=\left(x_{1}, y_{1}, z_{1}\right)$ and $\mathbf{r}_{2}=\left(x_{2}, y_{2}, z_{2}\right)$ correspond to the positions of the detectors and we have chosen experimental conditions such that $Z=z_{1}=z_{2}$. Here we are working in the paraxial and monochromatic approximations, and have assumed that the beam splitter is 50-50 and symmetric. Furthermore, we disregard any entanglement between the momentum and polarization degrees of freedom [29]. The $\left(-y_{1}-y_{2}\right) / 2$ term in the $\mathcal{W}$ functions are a consequence of the reflection of both fields at the beam splitter.

A quick look at (3) and (4) shows that when the pump beam profile is an even function of $y$, only $\left|\psi^{-}\right\rangle$gives coincidence counts at the outputs of the BS (fig. 1 a)). If the pump beam profile is an odd function of $y$, then $\left|\phi^{ \pm}\right\rangle$and $\left|\psi^{+}\right\rangle$give coincidence counts, while $\left|\psi^{-}\right\rangle$results in two photons in the same output of the beam splitter. Thus, using a pump beam profile that is an odd function of $y$ gives the desired interference effects for our BSM scheme mentioned above.

A well known class of beams with cartesian parity are 


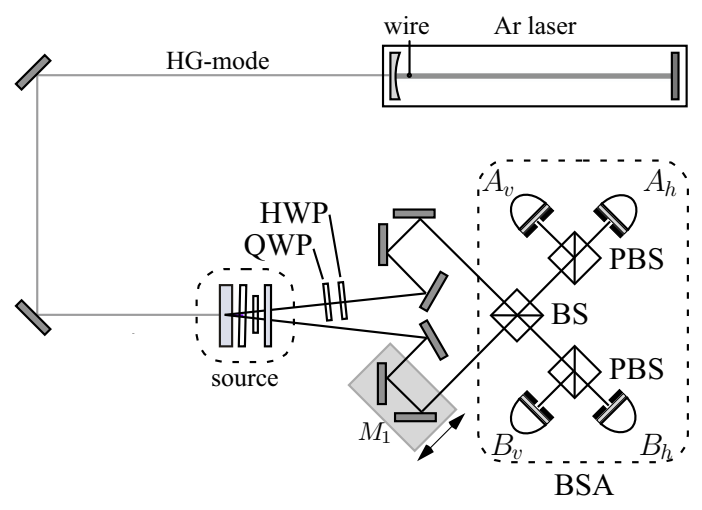

FIG. 2: Experimental setup. The source is described in the text. BSA is the Bell-state analyzer consisting of a beam splitter (BS) and two polarizing beam splitters (PBS).

the Hermite-Gaussian (HG) beams,

$$
\begin{aligned}
\mathrm{HG}_{m n}(x, y, z)= & C_{m n} H_{m}(x \sqrt{2} / w) H_{n}(y \sqrt{2} / w) \times \\
& e^{\left(x^{2}+y^{2}\right) / w^{2}} e^{-i k\left(x^{2}+y^{2}\right) / 2 R} e^{-i(m+n+1) \theta},
\end{aligned}
$$

where $C_{m n}$ is a normalization constant. The $H_{n}(y)$ are the Hermite polynomials, which are even or odd functions in the $y$-coordinate when the index $n$ is even or odd, respectively. $w$ is the beam radius, $R(z)=\left(z^{2}+z_{R}^{2}\right) / z$ and $\theta(z)=\arctan \left(z / z_{R}\right)$, where $z_{R}$ is the Rayleigh range. Using a pump beam in the $\mathrm{HG}_{01}$ mode, we can perform an incomplete Bell-state analysis in the coincidence basis.

The experiment is shown in fig. 2. An argon-ion laser $(351.1 \mathrm{~nm})$ is used to pump a $2 \mathrm{~mm}$ thick BBO crystal. The crystal is cut for type-II phase matching at $\lambda_{1}=\lambda_{2}=702.2 \mathrm{~nm}$. To generate a first-order HG mode, a $25 \mu \mathrm{m}$ diameter wire was placed inside the laser cavity. The wire breaks the cylindrical symmetry of the laser cavity, forcing the laser to operate in an HG mode with a nodal line at the position of the wire [28]. With the wire placed vertically, we were able to generate the mode $\mathrm{HG}_{01}$ with an output power of $\sim 30 \mathrm{~mW}$. The downconverted beams leave the crystal at angles of $\sim 3^{\circ}$ and pass through a half-wave plate followed by a $1 \mathrm{~mm}$ thick BBO compensating crystal, as described in 86. A UV mirror is used to reflect the pump beam. A half-wave plate (HWP) and quarter-wave plate (QWP) are used to select between the four Bell states [8]. Trombone mirror assemblies are used to direct the down-converted beams onto a 50-50 beam splitter (BS). The relative path length is adjusted by moving mirror assembly $M_{1}$ with a motorized translation stage. The BS is mounted on a translation stage and can be moved in and out of the downconverted beams. This allows for polarization analysis of the Bell-states without the BS. The polarization analyzers (not shown) are rotatable half-wave plates followed by polarizing beam splitters. Detectors $D_{1}$ and $D_{2}$ are avalanche photodiodes equiped with $1 \mathrm{~nm}$ FWHM
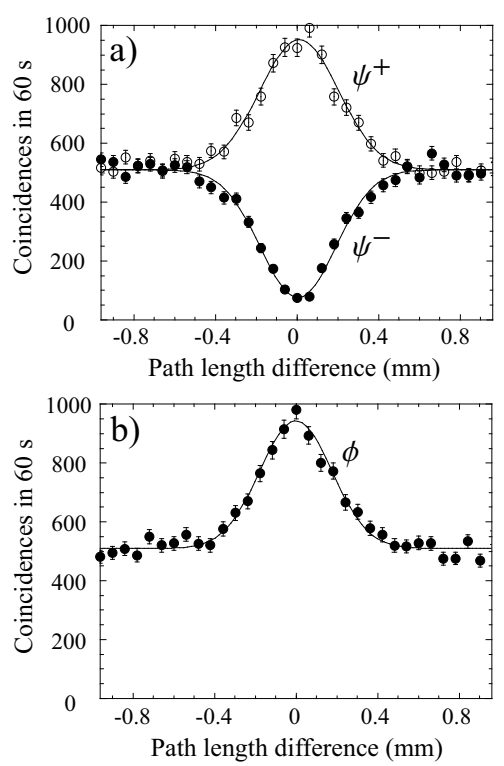

FIG. 3: Two-photon interference for a) $\left|\psi^{+}\right\rangle(\circ),\left|\psi^{-}\right\rangle(\bullet)$ and b) $|\phi\rangle$ when the pump beam is the Hermite-Gaussian mode $\mathrm{HG}_{01}$.

bandtwidth interference filters and $3 \mathrm{~mm}$ diameter collection apertures. Coincidence and single counts were registered by a personal computer.

Polarization analysis (BS and PBS removed) of the four Bell states generated with a $\mathrm{HG}_{01}$ pump beam gave interference curves (not shown) with visibilities $\sim 0.94-0.97$, which were comparable to results when a Gaussian pump beam was used. Since we are unable to distinguish between $\left|\phi^{+}\right\rangle$and $\left|\phi^{+}\right\rangle$with the BSA, we chose to define $\left|\phi^{+}\right\rangle \equiv|\phi\rangle$. Fig. 3 shows the HOM interference for the states $\left|\psi^{ \pm}\right\rangle$and $|\phi\rangle$ when the $\mathrm{HG}_{01}$ beam is used and the PBS were removed. Visibilities of $\sim 0.85 \pm 0.02$ were achieved with the $\mathrm{HG}_{01}$ mode, which were slightly lower than with a Gaussian pump beam $(\sim 0.92 \pm 0.01)$. This was most likely due to misalignment of the wire in the laser cavity, as well as an increased sensitivity to alignment of the interferometer with the $\mathrm{HG}_{01}$ pump beam. For the BSM, the mirror assembly was placed at position " 0 ". Comparing the interference maxima and minimum at position 0 with the constant count outside the interference region, there is a $\sim 94 \%$ probability that photons in $\left|\psi^{+}\right\rangle$and $|\phi\rangle\left(\left|\psi^{-}\right\rangle\right)$ end up in different (the same) outputs. Fig. 4 shows results of the incomplete BSM for the states $\left|\psi^{-}\right\rangle,\left|\psi^{+}\right\rangle$and $|\phi\rangle$. States were discriminated with a success probability of $\sim 91 \%$.

\section{COMPLETE BELL-STATE ANALYSIS IN THE COINCIDENCE BASIS}

In ref. [17], Kwiat and Weinfurter outline a scheme for complete Bell-state analysis using hyperentanglement, 

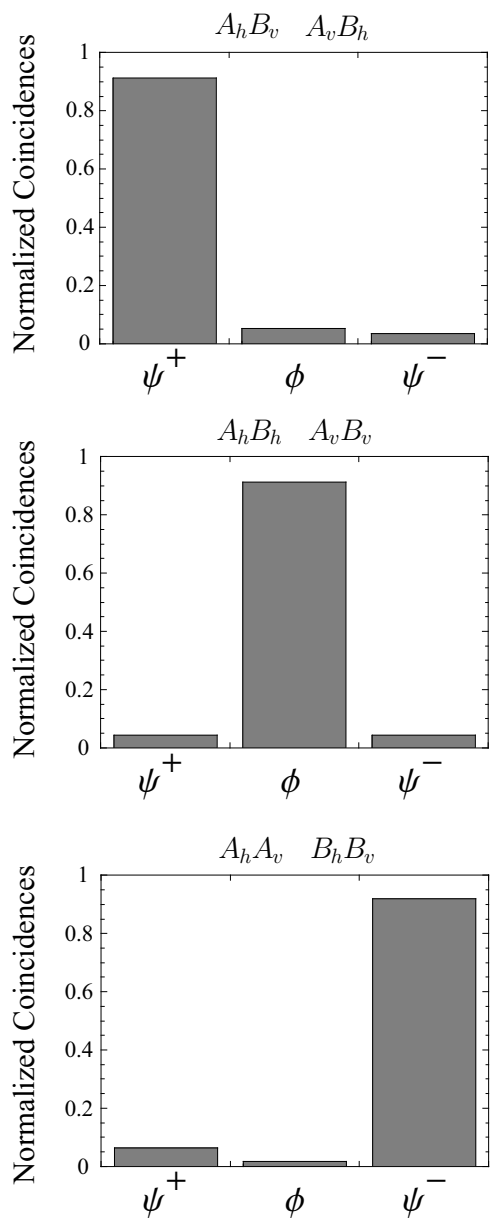

FIG. 4: Experimental results for incomplete Bell- state measurement $(\mathrm{BSM})$ in the coincidence basis for the three input states $\left|\psi^{+}\right\rangle,\left|\psi^{-}\right\rangle$and $|\phi\rangle$.

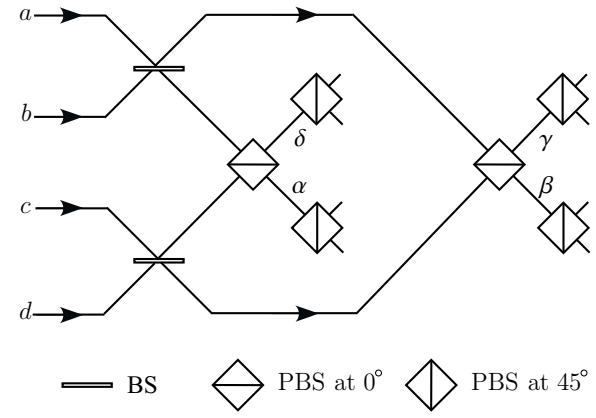

FIG. 5: Kwiat and Weinfurter's complete Bell-state analyzer using hyperentangled states 17].

shown in fig. 5. Using hyperentangled states of the form

$$
\begin{aligned}
\psi^{ \pm} & =\left\{\left(a_{h} b_{v} \pm a_{v} b_{h}\right)+\left(c_{h} d_{v} \pm c_{v} d_{h}\right)\right\} / 2, \\
\phi^{ \pm} & =\left\{\left(a_{h} b_{h} \pm a_{v} b_{v}\right)+\left(c_{h} d_{h} \pm c_{v} d_{v}\right)\right\} / 2,
\end{aligned}
$$

where $a_{h}\left(a_{v}\right)$ stands for a horizontally (vertically) polarized photon in mode $a$, the authors show that the final states are

$$
\begin{aligned}
\psi^{+}= & i\left(\alpha_{45} \delta_{45}-\alpha_{\overline{45}} \delta_{\overline{45}}+\beta_{45} \gamma_{45}-\beta_{\overline{45}} \gamma_{\overline{45}}\right) / 2 \\
\psi^{-}= & \left(\alpha_{45} \gamma_{45}-\alpha_{\overline{45}} \gamma_{\overline{45}}+\beta_{45} \delta_{45}-\beta_{\overline{45}} \delta_{\overline{45}}\right) / 2 \\
\phi^{-}= & -i\left(\alpha_{45} \alpha_{\overline{45}}-\beta_{45} \beta_{\overline{45}}+\gamma_{45} \gamma_{\overline{45}}-\delta_{45} \delta_{\overline{45}}\right) / 2 \\
\phi^{+}= & i\left(\alpha_{45} \alpha_{45}+\alpha_{\overline{45}} \alpha_{\overline{45}}+\beta_{45} \beta_{45}+\beta_{\overline{45}} \beta_{\overline{45}}+\gamma_{45} \gamma_{45}\right. \\
& \left.+\gamma_{\overline{45}} \gamma_{\overline{45}}+\delta_{45} \delta_{45}+\delta_{\overline{45}} \delta_{\overline{45}}\right) /(2 \sqrt{2})
\end{aligned}
$$

where $\overline{45}$ is polarization orthogonal to 45 . Each of the four states above gives a different signature of detectors firing, however, a detector capable of distinguishing between one and two photons is required to detect $\phi^{+}$.

We wish to show how the use of an antisymmetric pump beam can improve on these results. Using an antisymmetric pump beam such as the first-order Hermite Gaussian beam $H G_{01}$, it is easy to show that after the first set of beam splitters (BS), the states (6), (7) are

$$
\begin{aligned}
& \psi^{+}=\left\{\left(a_{h} b_{v}+a_{v} b_{h}\right)+\left(c_{h} d_{v}+c_{v} d_{h}\right)\right\} / 2, \\
& \psi^{-}=i\left\{\left(a_{h} a_{v}-b_{v} b_{h}\right)+\left(c_{h} c_{v}-d_{v} d_{h}\right)\right\} / 2, \\
& \phi^{ \pm}=\left\{\left(a_{h} b_{h} \pm a_{v} b_{v}\right)+\left(c_{h} d_{h} \pm c_{v} d_{v}\right)\right\} / 2 .
\end{aligned}
$$

After the polarizing beam splitters (PBS) at $0^{\circ}$ and $45^{\circ}$, these states become

$$
\begin{aligned}
& \psi^{+}=\left(\alpha_{45} \gamma_{45}-\alpha_{\overline{45}} \gamma_{\overline{45}}+\beta_{45} \delta_{45}-\beta_{\overline{45}} \delta_{\overline{45}}\right) / 2, \\
& \psi^{-}=i\left(\beta_{45} \gamma_{45}-\beta_{\overline{45}} \gamma_{\overline{45}}+\alpha_{45} \delta_{45}-\alpha_{\overline{45}} \delta_{\overline{45}}\right) / 2, \\
& \phi^{-}=\left(\alpha_{45} \beta_{\overline{45}}+\alpha_{\overline{45}} \beta_{45}+\gamma_{45} \delta_{\overline{45}}+\gamma_{\overline{45}} \delta_{45}\right) / 2, \\
& \phi^{+}=\left(\alpha_{45} \beta_{45}+\alpha_{\overline{45}} \beta_{\overline{45}}+\gamma_{45} \delta_{45}+\gamma_{\overline{45}} \delta_{\overline{45}}\right) / 2 .
\end{aligned}
$$

Each of these states has its own signature of detectors firing in coincidence.

\section{CONCLUSION}

We have shown how Hong-Ou-Mandel interference at a beam splitter can be controlled to facilitate Bell-state analysis. Creating polarization-entangled photons with spontaneous parametric down-conversion using an antisymmetric Hermite-Gaussian pump beam, we invert the usual interference behavior of the Bell states. We have shown how that this simplifies the standard methods for incomplete Bell-state analysis of down-converted photon pairs, removing the necessity for detectors sensitive to photon number. In addition, we have shown that we can improve on a previous scheme for complete Bellstate analysis of down-converted photon pairs using hyperentanglement 17, enabling complete Bell-state analysis in the coincidence basis. These results illustrate the use of additional degrees of freedom of the two-photon state as a control parameter in quantum state engineering.

It is important to note that these results are applicable for down-converted pairs only, and thus are not entirely helpful for BSM's in quantum teleportation [4] 
or entanglement swapping [6, 7] protocol which use photon pairs which are not created simultaneously from a common source. However, we emphasize that our results are directly applicable to quantum dense coding schemes [1, 2.

\section{Acknowledgments}

The authors thank the Brazilian funding agencies CNPq and CAPES.
[1] C. Bennett and S. Weisner, Phys. Rev. Lett. 69, 2881 (1992).

[2] K. Mattle, H. Weinfurter, P. Kwiat, and A. Zeilinger, Phys. Rev. Lett. 76, 4656 (1996).

[3] C. Bennett, G. Brassard, C. Crépeau, R. Jozsa, A. Peres, and W. Wooters, Phys. Rev. Lett. 70, 1895 (1993).

[4] D. Bouwmeester, J. Pan, K. Mattle, M. Eibl, H. Weinfurter, and A. Zeilinger, Nature 390, 575 (1997).

[5] D. Boschi, S. Branca, F. DeMartini, L. Hardy, and S. Popescu, Phys. Rev. Lett. 80, 1121 (1998).

[6] J.-W. Pan, D. Bouwmeester, H. Weinfurter, and A. Zeilinger, Phys.Rev. Lett. 80, 3891 (1998).

[7] T. Jennewein, G. Weihs, J.-W. Pan, and A. Zeilinger, Phys. Rev. Lett. 88, 017903 (2002).

[8] P. G. Kwiat, K. Mattle, H. Weinfurter, A. Zeilinger, A. V. Sergienko, and Y. Shih, Phys. Rev. Lett. 75, 4337 (1995).

[9] P. G. Kwiat, E. Waks, A. G. White, I. Appelbaum, and P. H. Eberhard, Phys. Rev. A. 60, R773 (1999).

[10] L. Vaidman and N. Yoran, Phys. Rev. A 59, 116 (1999).

[11] N. Lütkenhaus, J. Calsamiglia, and K.-A. Suominen, Phys. Rev. A 59, 3295 (1999).

[12] J. Calsamiglia and N. Lütkenhaus, Appl. Phys. B 72, 67 (2001).

[13] S. Ghosh, G. Kar, A. Roy, A. Sen(De), and U. Sen, Phys. Rev. Lett. 87, 277902 (2001).

[14] Y.-H. Kim, S. P. Kulik, and Y. H. Shih, Phys. Rev. Lett. 86, 1370 (2001).

[15] S. Braunstein and A. Mann, Phys. Rev. A. 51, R1727 (1995).
[16] M. O. Scully, B.-G. Englert, and C. J. Bednar, Phys. Rev. Lett. 83, 4433 (1999).

[17] P. G. Kwiat and H. Weinfurter, Phys. Rev. A 58, R2623 (1998).

[18] M. Michler, K. Mattle, H. Weinfurter, and A. Zeilinger, Phys. Rev. A. 53, R1209 (1996).

[19] H. Weinfurter, Europhys. Lett. 25, 559 (1994).

[20] C. K. Hong, Z. Y. Ou, and L. Mandel, Phys. Rev. Lett. 59, 2044 (1987).

[21] A. Zeilinger, H. J. Bernstein, and M. A. Horne, J. Mod. Optics 41, 2375 (1994).

[22] P. G. Kwiat, A. M. Steinberg, R. Y. Chiao, P. H. Eberhard, and M. D. Petroff, Phys.Rev. A 48, R867 (1993).

[23] J. Kim, S. Takeuchi, Y. Yamamoto, and H. H. Hogue, Appl. Phys. Lett. 74, 902 (1999).

[24] S. Takeuchi, J. Kim, Y. Yamamoto, and H. H. Hogue, Appl. Phys. Lett. 74, 1063 (1999).

[25] S. P. Walborn, A. N. de Oliveira, S. Pádua, and C. H. Monken (2002), quant-ph/0212017.

[26] C. H. Monken, P. S. Ribeiro, and S. Pádua, Phys. Rev. A. 57, 3123 (1998).

[27] C. K. Hong and L. Mandel, Phys. Rev. A 31, 2409 (1985).

[28] M. W. Beijersbergen, L. Allen, H. E. L. O. van der Veen, and J. P. Woerdman, Optics Comm. 96, 123 (1993).

[29] Entanglement between wave vector and polarization can be made negligible by using compensating crystals as well as small detection irises and narrow bandwidth interference filters. 